\title{
Adenovirus-mediated p53 and ING4 gene co-transfer elicits synergistic antitumor effects through enhancement of p53 acetylation in breast cancer
}

\author{
$\mathrm{JIE} \mathrm{WU}^{1 *}$, YANBO ZHU $^{1 *}, \mathrm{CHUN} \mathrm{XU}^{1}, \mathrm{HONG} \mathrm{XU}^{1}, \mathrm{XIUMIN} \mathrm{ZHOU}^{1}, \mathrm{JICHENG} \mathrm{YANG}^{2}$, \\ YUFENG XIE $^{1}$ and MIN TAO ${ }^{1}$ \\ ${ }^{1}$ Department of Oncology, The First Affiliated Hospital of Soochow University, Suzhou, Jiangsu; \\ ${ }^{2}$ Department of Cell Biology, College of Medicine, Soochow University, Suzhou, Jiangsu, P.R. China
}

Received July 26, 2015; Accepted September 17, 2015

DOI: $10.3892 /$ or.2015.4385

\begin{abstract}
Multigene-based combination therapy may be an effective practice in cancer gene therapy. Substantial studies have demonstrated that tumor suppressor p53 acetylation is indispensable for p53 activation. Inhibitor of growth 4 (ING4), as a novel tumor suppressor, is capable of remarkably enhancing p53 acetylation and its transcriptional activity. Hence, we assumed that combined treatment of p53 and ING4 double tumor suppressors would exhibit enhanced antitumor effects. The combined therapeutic efficacy of p53 and ING4 for human cancers has not been previously reported. We thus generated multiple promoter expression cassette-based recombinant adenovirus-co-expressing ING4 and p53 double tumor suppressor genes (AdVING4/p53), evaluated the combined effects of AdVING4/p53 on breast cancer using the MDA-MB-231 (mutant p53) human breast cancer cell line, and also elucidated its underlying molecular mechanisms. We demonstrated that AdVING4/p53-mediated p53 and ING4 co-expression induced synergistic growth inhibition and apoptosis as well as enhanced effects on upregulation of acetylated p53, P21, Bax, PUMA, Noxa, cleaved caspase-9, cleaved caspase-3 and cleaved PARP, and downregulation of Bcl-2, CD31 and microvessel density (MVD) in MDA-MB231 breast cancer in vitro and/or in vivo subcutaneous (s.c.) xenografted tumors. The synergistic antitumor activity elicited by AdVING4/p53 was closely associated with the enhanced activation of the intrinsic apoptotic pathway and synergistic
\end{abstract}

Correspondence to: Dr Min Tao or Professor Yufeng Xie, Department of Oncology, The First Affiliated Hospital of Soochow University, Suzhou, Jiangsu 215006, P.R. China

E-mail: mtao@medmail.com.cn

E-mail: sdxyf@163.com

*Contributed equally

Key words: p53, inhibitor of growth 4, breast cancer, combined gene therapy, synergistic antitumor effect inhibition of tumor angiogenesis, very possibly via ING4mediated enhancement of p53 acetylation and activity. Thus, our results indicate that cancer gene therapy combining two or more tumor suppressors such as p53 and ING4 may constitute a novel and effective therapeutic modality for human breast cancer and other cancers.

\section{Introduction}

The p53 tumor suppressor is a central 'cellular gatekeeper', also referred to as 'the guardian of the genome', which is critically implicated in monitoring diverse cellular processes including cell cycle arrest, DNA repair, senescence, apoptosis, differentiation, cell metabolism and autophagy under various cellular stresses (1). p53 as a transcription factor can bind to the promoter regions of hundreds of target genes and regulate their transcription to elicit its functions (2). The well-described p53 target genes include P21 involved in cell cycle arrest, Bax, Puma and Noxa involved in apoptosis, Nanog involved in differentiation, and PAI-1 involved in senescence (2). The p53 gene is commonly mutated in 50\% of all human cancers (3). Somatic p53 missense mutations discrupt the p53 binding to DNA response elements leading to the repression of target gene transcription. Loss of wildtype p53 function is crucial for human cancer progression. In addition to the loss-of-function or dominant-negative effect, many mutant p53 proteins acquire tumor-promoting functions via multiple mechanisms (4,5). Moreover, many cancers expressing mutant p53 increases resistance to chemotherapy and radiotherapy (6). Even in human cancers that bear wildtype $\mathrm{p} 53$, the $\mathrm{p} 53$ upstream or downsteam regulatory pathways as well as post-translational modifications are disrupted, suggesting that essentially all cancers have alterations in the p53 pathway (7). Based upon powerful tumor-suppressive effects of p53, restoration of wild-type p53 function is thus an attractive therapeutic approach for the treatment of human cancers. The strategies to restore wild-type p53 function consist of wild-type p53 gene therapy, activation of endogenous wild-type p53 using SIRT1/2 inhibitor tenovins, nuclear export inhibitor Crm1 and MDM2 inhibitors, reactivation of mutant p53 using peptides or compounds, and activating other 
p53 family member p73 (8). Among them, adenovirus-based p53 cancer gene therapy such as Advexin and Gendicine has been tested in clinical trials.

The inhibitor of growth 4 (ING4) is a novel tumor suppressor belonging to type II tumor suppressor family including ING1-ING5, the five known members (9). ING4 has been found to be commonly deleted, mutated or downregulated in a large variety of cancers, which contributes to tumorigenesis, cancer development and prognosis (9). Accumulating evidence has shown that ING4 can induce tumor growth suppression via induction of cell cycle alteration, apoptosis and toxic autophagy in a p53-dependent or -independent fashion in a broad spectrum of cancer cells (10-15). Notably, ING4 enhances the sensitivity of chemotherapy, intracavitary radiotherapy or external beam radiotherapy in heptocarcinoma $(11,16)$, pancreatic cancer (17) and lung cancer (18). ING4 also suppresses oncogenes such as myelocytomatosis viral related oncogene, neuroblastoma derived (MYCN)- and myelocytomatosis viral oncogene homolog (MYC)-induced loss of contact inhibition (19). Furthermore, ING4 represses cancer spreading, migration and invasion by interacting with liprin $\alpha 1$ at lamellipodia (20) and downregulating matrix metalloproteinase (MMP)-2 and MMP-9 $(13,21)$. Additionally, ING4 downregulates the expression of IL-6 and IL-8 pro-angiogenic factors and consequently suppresses tumor angiogenesis via repressing transcription activity of nuclear factor- $\kappa \mathrm{B}(\mathrm{NF}-\kappa \mathrm{B})(22,23)$ and hypoxia inducible factor-1 $\alpha$ (HIF-1 $\alpha)(24,25)$ in a chromatin-remolding manner. Interestingly, ING4 can act as a novel E3 ubiquitin ligase that directly induces NF- $\kappa \mathrm{B}$ p65 degradation and consequently terminates NF- $\kappa B$ activation (26). More recently, ING4 has been shown to interact with AUF1 and reduce pro-oncogene c-myc translational expression (27). These findings revealed that ING4 as a promising candidate tumor suppressor negatively regulating cancer growth via targeting multiple pathways.

Breast cancer is the most frequently diagnosed malignancy among women and the first leading cause of cancer-related death in female worldwide, accounting for $23 \%$ of the total new cases and $14 \%$ of the cancer deaths (28). Conventional therapies for breast cancer such as surgery, chemotherapy, radiation therapy, hormone therapy and targeted therapy have demonstrated a considerable clinical success over the past few years. However, de novo and acquired resistance is one of the major obstacles in treatment of breast cancer, warranting the search for new therapeutic alternatives for breast cancer. Gene therapy is considered as a promising therapeutic modality for human cancers (29). Combined gene therapy may be an effective practice in cancer gene therapy, which can achieve greater therapeutic benefit by targeting multiple pathways $(29,30)$. Previous studies showed that tumor suppressor p53 acetylation is indispensable for p53 activation (31). Previous studies also showed that tumor suppressor ING4 can physically interact with p300 and p53, thereby enhancing p53 acetylation and its transcriptional activity (10). In the present study, we thus investigated whether adenovirus-mediated p53 and ING4 combined gene therapy would produce enhanced antitumor effects in MDA-MB-231 (mutant p53) human breast cancer in vitro and in vivo in an athymic nude mouse model, and also elucidated its molecular mechanisms.

\section{Materials and methods}

Vectors, cell lines, reagents and mice. The polyA+hEF1a-eIF4g promoter expression cassette-modified adenoviral transfer plasmid pAdTrack-CMV/polyA+hEF1a-eIF4g with three independent promoters including two cytomegalovirus (CMV) promoters driving the interesting gene (1st CMV) and green fluorescent protein (GFP) maker gene (2nd CMV) expression, respectively; and one inserted hEF1a-eIF4g hybrid promoter driving the other interesting gene expression were constructed in our laboratory (32). The pAdTrack-CMV/ING4 carrying humanized ING4 coding sequence was also constructed in our laboratory (13). The human wild-type p53 open reading frame (ORF) clone was purchased form Sino Biological Inc. (Beijing, China). The integrin-binding motif Arg-Gly-Asp (RGD)-modified pAdEasy-1 (Ad5E1/E3-deleted) adenoviral backbone plasmid (33) was kindly provided by Professor Jim Xiang (Saskatoon Cancer Agency, Saskatoon, SK, Canada). The BJ5183 bacteria and QBI-293A human embryonic kidney cell line for adenovirus packaging and amplification were kindly provided by Professor Jiang Zhong (Department of Microbiology, College of Life Science, Fudan University, Shanghai, China). The MDA-MB-231 (mutant p53, R280K) (4) human breast cancer cell line was purchased from the Cell Bank, Type Culture Collection of Chinese Academy of Sciences (Shanghai, China). The Dulbecco's modified Eagle's medium (DMEM) and fetal bovine serum (FBS) were purchased from Hyclone (Logan, UT, USA). The MiniBEST Universal RNA extraction kit was purchased from Takara (Dalian, Liaoning, China). The reverse transcriptase polymerase MuMLV and OligodT18 were purchased from Thermo Fisher Scientific (Shanghai, China). The 3-(4,5-dimethylthiazol-2-yl)-2,5-diphenyltetrazolium bromide (MTT) and mammalian cell lysis kit were purchased from Sigma (Shanghai, China). The Annexin V-PE/7-AAD apoptosis detection kit was purchased from BD Biosciences (Shanghai, China). The antibodies specific for ING4, p53, Ac-p53 (K382), P21, Bax, PUMA, Noxa, Bcl-2, caspase-9, caspase-3, poly(ADP-ribose) polymerase (PARP), $\beta$-actin and CD31 were purchased from Santa Cruz Biotechnology (Shanghai, China) and Cell Signaling Technology (Danvers, MA, USA). The SuperEnhanced chemiluminescence detection kit was purchased from Applygen Technology Inc. (Beijing, China). The terminal deoxynucleotidyl transferase-mediated dUTP nick end labeling (TUNEL) apoptosis detection kit was purchased from Beyotime Biotechnology (Beijing, China). The UltraSensitive $^{\mathrm{TM}}$ SP kit was purchased from Maixin (Fuzhou, Fujian, China). The primers were synthesized from Sangon Biotechnology Inc. (Shanghai, China). The 4-week-old female athymic BALB/c nude mice were purchased from Shanghai Experimental Animal Center (Shanghai, China) and maintained in the animal facility at Soochow University (Suzhou, China) according to the Animal Research Committee guidelines of Soochow University.

Construction of an adenovirus co-expressing ING4 and p53 tumor suppressor genes. We previously generated two kinds of recombinant adenovirus co-expressing ING4 and interleukin (IL)-24 using polyA+hEF1a-eIF4g promoter expression cassette and internal ribosome entry site (IRES) element, 
respectively (32). We demonstrated that inserted hEF1a-eIF4g promoter more efficiently directed the translational expression of downstream gene IL-24 than IRES element (32). In the study, we thus constructed an adenovirus co-expressing ING4 and p53 double tumor suppressor genes using polyA+hEF1a-eIF4g promoter expression cassette modality (Fig. 1A). Briefly, the ING4 and p53 coding sequence (CDS) fragments were amplified by PCR using pAdTrackCMV/ING4 plasmid (13) or p53 ORF clone plasmid as a template and primers specific for human ING4 (ING4 forward, 5'-tag aga tct acc atg get get ggg atg tat ttg g-3' and reverse, 5 '-acc gtc gac cet att tct tct tcc gtt ctt g-3') or p53 (p53 forward, 5'-gca ctc gag acc atg gag gag ccg cag tca gat c-3' and reverse, 5 '-gct tct aga tca gtc tga gtc agg ccc ttc-3') as primers, and then subcloned into pAdTrack-CMV/polyA+hEF1a-eIF4g modified transfer plasmid (32) at BglII, SalI; XhoI, XbaI sites, respectively. To develop a more efficient gene delivery system, we used Arg-Gly-Asp (RGD)-modified pAdEasy-1 (Ad5E1/E3-deleted) that contains an integrin-binding motif RGD sequence in the HI loop of adenoviral fiber as an adenoviral backbone plasmid (33). After its homologous recombination with RGD-modified pAdEasy-1 in BJ5183 bacteria, the replication-incompetent and RGD-modified adenovirus AdVING4/p53 expressing GFP marker gene was subsequently produced and abundantly amplified in QBI-293A cells as described previously (34), and then purified by cesium chloride $(\mathrm{CsCl})$ density-gradient ultracentrifugation. The adenoviruses such as AdVING4, AdVp53, AdV used for controls were also similarly prepared.

Fluorescence microscopic analysis. The titer of AdVING4/p53, AdVING4, AdVp53 and AdV adenoviruses was determined using gene transfer unit (GTU) method by calculating the number of GFP-expressing QBI-293A cells within $18 \mathrm{~h}$ after adenoviral infection under fluorescence microscopy. Accordingly, the ratio of infectious adenovirus (GTU) to target cells is called multiplicity of infection (MOI). To select an optimal MOI for a maximal adenoviral infection and human ING4/p53 transgene expression in MDA-MB-231 tumor cells, the MDA-MB-231 human breast cancer cells ( $1 \times 10^{4}$ cells) were treated with AdVING4/p53, AdVING4, AdVp53, AdV or PBS without adenovirus at various MOIs $(0,10,25,50,100$ or 200). Twenty-four hours after infection, the GFP expression and adenoviral infection efficiency was observed by fluorescence microscopy.

Reverse transcription (RT)-PCR analysis. The adenovirus-mediated human ING4/p53 transcriptional expression was analyzed by reverse transcription (RT)-PCR. The MDA-MB-231 human breast cancer cells $\left(2 \times 10^{6}\right.$ cells $)$ were treated with AdVING4/p53 (50 MOI), AdVING4 (50 MOI), AdVp53 (50 MOI), AdV (50 MOI) or PBS without adenovirus for $24 \mathrm{~h}$. Total cellular RNAs were purified from AdVING4/p53-, AdVING4-, AdVp53-, AdV-infected MDA-MB-231 tumor cells or uninfected control cells and the first-strand cDNAs were synthesized from RNAs using reverse transcriptase MuMLV. The PCR reactions were then carried out using cDNA as a template and primers specific for human ING4 and p53 described above, or housekeep gene $\beta$-actin ( $\beta$-actin forward, 5'-tgc gtg aca tta agg aga ag-3' and reverse,
5 '-ctg cat cct gtc ggc aat g-3') as primers. The reaction products were analyzed on $1 \%$ agarose gel electrophoresis with ethidium bromide staining.

Cell viability assay. The effect of AdVING4/p53 on the MDA-MB-231 human breast cancer cell growth in vitro was assessed by MTT assay. Briefly, the MDA-MB-231 tumor cells were dispensed into 96 -well plates at $1 \times 10^{4}$ cells/well/200 $\mu \mathrm{l}$ culture medium, i.e. DMEM supplemented with $10 \%$ FBS. After $24 \mathrm{~h}$ of incubation, the MDA-MB-231 tumor cells were infected with AdVING4/p53, AdVING4, AdVp53 or AdV at the optimal MOI of 50 in culture medium and treated for the indicated time periods. The medium containing PBS without adenovirus was used as a cell control (PBS control). Before infection (day 0) and at days 1, 2, 3 and 4 after infection, the viability of MDA-MB-231 tumor cells was analyzed using MTT kit according to the manufacturer's protocols.

Flow cytometric analysis. The effect of AdVING4/p53 on MDA-MB-231 human breast cancer cell apoptosis in vitro was evaluated by flow cytometric analysis with Annexin V-PE (early apoptotic marker) and 7-AAD (late apoptotic marker) double staining according to the company protocols. Briefly, the MDA-MB-231 tumor cells $\left(1 \times 10^{6}\right.$ cells) were treated with AdVING4/p53 (50 MOI), AdVING4 (50 MOI), AdVp53 (50 MOI), AdV (50 MOI) or PBS without adenovirus in culture medium for $24 \mathrm{~h}$. The uninfected and AdVING4/p53-, AdVING4-, AdVp53- or AdV-infected MDA-MB-231 tumor cells were harvested and washed with cold PBS. The above tumor cells $\left(1 \times 10^{5}\right.$ cells) were then incubated in the presence of $5 \mu \mathrm{l}$ Annexin V-PE and 7-AAD in $100 \mu \mathrm{l}$ of $1 \mathrm{X}$ Annexin V binding buffer at room temperature. After $15 \mathrm{~min}$ incubation, $400 \mu \mathrm{l}$ of $1 \mathrm{X}$ binding buffer was added and the apoptotic cells were analyzed by flow cytometry.

Western blot analysis. The MDA-MB-231 human breast cancer cells $\left(1 \times 10^{7}\right.$ cells) were treated with AdVING4/p53 (50 MOI), AdVING4 (50 MOI), AdVp53 (50 MOI), AdV (50 MOI) or PBS without adenovirus. After $24 \mathrm{~h}$ of treatment, the AdVING4/p53-, AdVING4-, AdVp53-, AdV-infected MDA-MB-231 tumor cells or uninfected control cells were collected, washed with cold PBS and lysed in lysis buffer $\left(1 \times 10^{7}\right.$ cells $/ 1 \mathrm{ml}$ lysis buffer) for preparation of total cellular lysates using mammalian cell lysis kit following the kit instructions. The protein concentration was determined by BCA protein assay using a spectrophotometer. The total cellular lysates $(100 \mu \mathrm{g} / \mathrm{lane})$ were then subjected to $12 \%$ sodium dodecyl sulfate-polyacrylamide gel electrophoresis (SDS-PAGE) and western blot analysis using a panel of primary antibodies specific for human ING4, p53, Ac-p53 (K382), P21, Bax, PUMA, Noxa, Bcl-2, caspase-9, caspase-3, PARP and $\beta$-actin following by corresponding horseradish peroxidase (HRP)-conjugated secondary antibody, respectively. After washing the membrane was developed using SuperEnhanced chemiluminescence detection kit. The bands were visualized after exposure of the membrane to Kodak X-ray film.

Animal study. The female athymic BALB/c nude mice were subcutaneously (s.c.) implanted with $2 \times 10^{6}$ MDA-MB-231 human breast cancer cells and monitored daily for tumor 
A

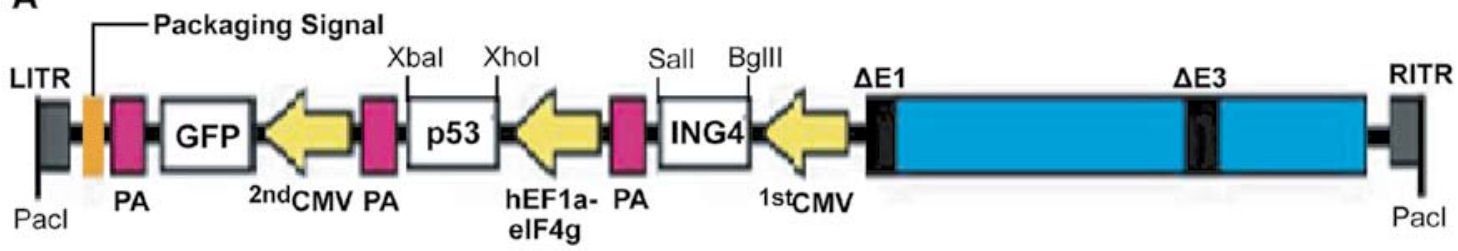

B
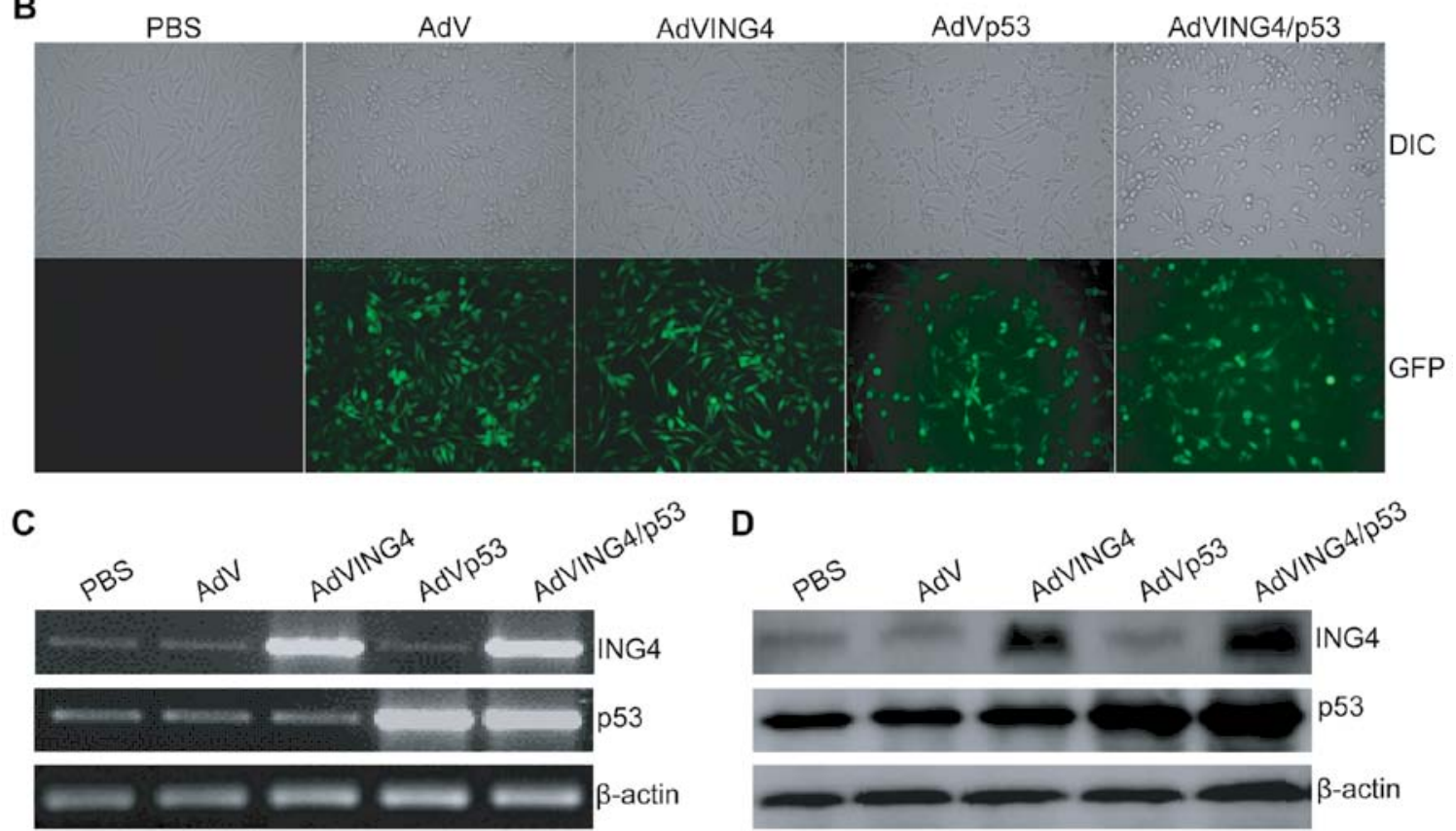

Figure 1. Adenovirus-mediated ING4 and p53 co-expression in breast cancer. (A) The construction strategy of an adenovirus co-expressing ING4 and p53 (AdVING4/p53). The ING4 and p53 CDS fragments were subcloned into pAdTrack-CMV/polyA+hEF1a-eIF4g modified adenoviral transfer plasmid at $B g l \mathrm{II}$, SalI; XhoI, XbaI sites, where ING4 transcription is under control of CMV (1st CMV) promoter, while p53 transcription is under control of hEF1a-eIF4g promoter. The AdVING4/p53 adenovirus was then generated by its homologous recombination with a RGD-modified pAdEasy-1 adenoviral backbone plasmid in BJ5183 bacteria followed by packaging and amplification in QBI-293A cells. CMV, cytomegalovirus promoter; hEF1a-eIF4g, hybrid promoter coupled 5 '-untranslated region (UTR) of the translation initiation factor eIF4g to elongation factor $1 \alpha$ (EF1 $\alpha$ ) promoter; GFP, green fluorescent protein; PA, SV40 polyadenylation signal; LITR, left-hand inverted terminal repeat; RITR, right-hand inverted terminal repeat. (B) Representative microphotos of differential interference contrast (DIC) and GFP. The MDA-MB-231 human breast cancer cells were infected with AdVING4/p53, AdVING4, AdVp53 or AdV at a MOI of 50 for $24 \mathrm{~h}$, and then observed under DIC and GFP fluorescence images by fluorescence microscopy. (C) RT-PCR analysis of adenovirus-mediated ING4 and p53 transcriptional expression. Total cellular RNAs were extracted from AdVING4/p53-, AdVING4-, AdVp53- or AdV-infected and uninfected MDA-MB-231 human breast cancer cells. The first-strand cDNAs were synthesized from RNAs using reverse transcriptase; PCRs were conducted using primer sets specific for human ING4, p53 and housekeep gene $\beta$-actin (an internal control), respectively. (D) Western blot analysis of adenovirus-mediated ING4 and p53 translational expression. Total cellular lysates derived from AdVING4/p53-, AdVING4-, AdVp53- or AdV-infected and uninfected MDA-MB-231 tumor cells were immunoblotted with anti-ING4, p53 and $\beta$-actin antibody, respectively. Data shown are representative of three independent experiments.

growth. Tumor volume was measured with a caliper and calculated by the following formula: tumor size $=a b^{2} / 2$, where $a$ is the larger of the two dimensions and $b$ is the smaller. When the tumors grew to a mean tumor volume of around $100 \mathrm{~mm}^{3}$, MDA-MB-231 human breast cancer s.c. xenografted tumor-bearing mice were subjected to gene therapy ( 5 mice each group) by multi-point intratumoral (i.t.) injection of AdVING4/p53 (1 $10^{8}$ GTU), AdVING4 (1x108 GTU), AdVp53 ( $\left.1 \times 10^{8} \mathrm{GTU}\right), \mathrm{AdV}\left(1 \times 10^{8} \mathrm{GTU}\right)$ or PBS every other day for a total of 5 times. Tumor progression was monitored and tumor volume was measured. The tumor-bearing mice were sacrificed 2 weeks after treatment. The xenografted tumors were then removed, weighted, fixed by $10 \%$ neutral formalin and embedded in paraffin for hematoxylin and eosin (H\&E) staining and immunohistochemistry analysis.

Immunohistochemistryanalysis. The expression of p53, Ac-p53 (K382), P21, Bax, PUMA, Noxa, Bcl-2, cleaved caspase-3 and
CD31 in MDA-MB-231 human breast cancer s.c. xenografted tumors was examined by immunohistochemistry analysis using UltraSensitive ${ }^{\mathrm{TM}}$ SP kit. The integral optical density (IOD) of immunohistochemical staining was quantitatively assessed by Image-Pro Plus 6.0 software (Media Cybernetics, Bethesda, MD, USA). Microvessel density (MVD) was determined by CD31 immunostaining. Any endothelial cell cluster immunoreactive for CD31 clearly separated from adjacent microvessels was considered as a single countable vessel. To detect the apoptotic cells in vivo in MDA-MB-231 xenografted tumors, tumor sections were further analyzed for apoptosis using TUNEL apoptosis detection kit following manufacturer's instructions. The IOD, MVD and TUNEL-positive cells was counted at 5 randomly selected high-power (x200 magnification) fields of each section by microscopy.

Statistical analysis and evaluation of combinatorial interaction. All data are presented as the mean \pm standard deviation 
A

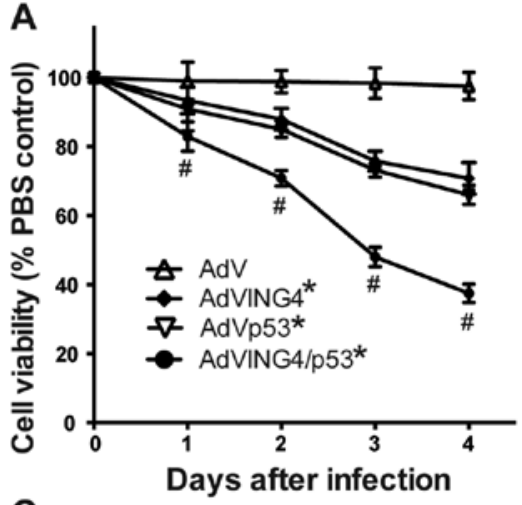

C

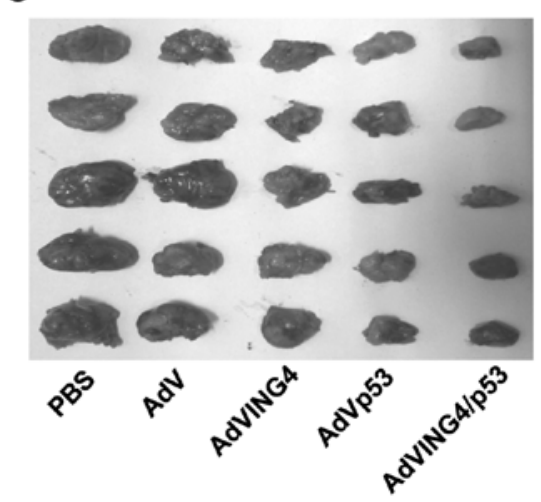

B

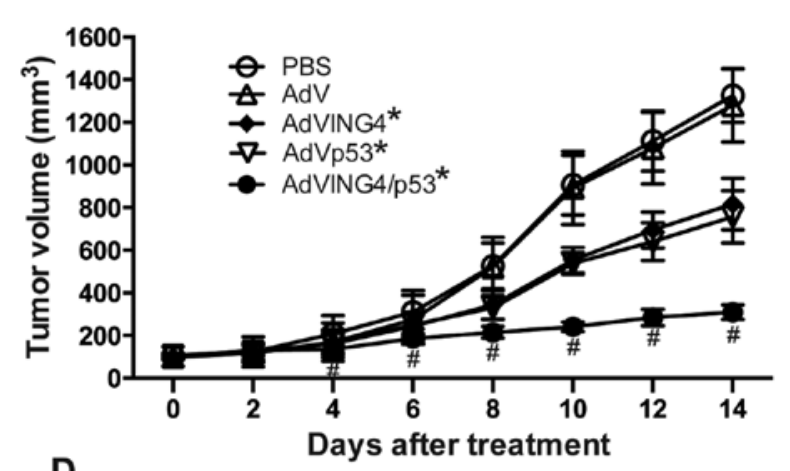

D

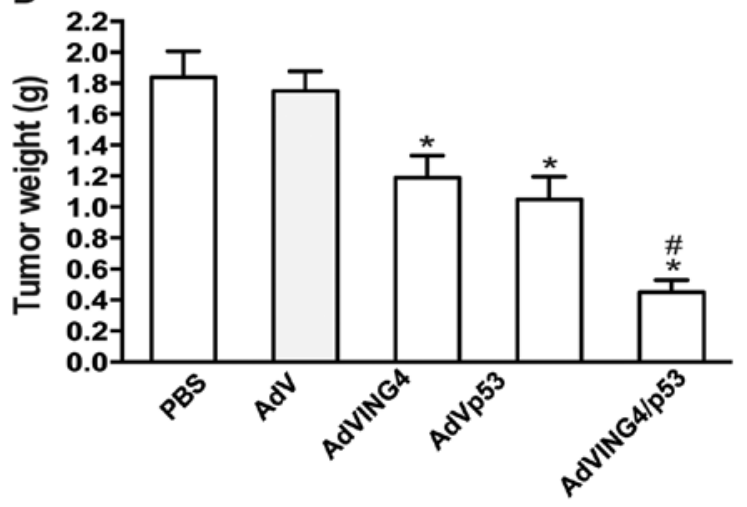

Figure 2. AdVING4/p53 induces synergistic tumor suppression in breast cancer. (A) AdVING4/p53 synergistically inhibits breast cancer growth in vitro. The MDA-MB-231 human breast cancer cells were treated with AdVING4/p53 (50 MOI), AdVING4 (50 MOI), AdVp53 (50 MOI), AdV (50 MOI) or PBS without adenovirus for the indicated time periods (0-4 days). The survival cells were evaluated at days $0,1,2,3$ and 4 after treatment by MTT assay. Tumor viability in vitro was calculated by comparison with PBS-treated control group according to absorbance value (OD $570 \mathrm{~nm}$ ). ${ }^{*} \mathrm{P}<0.05$ compared with PBS and AdV group; ${ }^{\mathrm{P}}<0.05$ compared with AdVING4 and AdVp53 group, $\mathrm{Q}=1.189,1.158,1.168$ and 1.176 at days 1, 2, 3 and 4 after treatment, respectively, two-way repeated measures ANOVA and multiple comparisons; $\mathrm{n}=4$ replicates per condition. (B-D) AdVING4/p53 synergistically inhibits breast cancer growth in vivo. The athymic BALB/c nude mice bearing MDA-MB-231 s.c. xenografted tumors were i.t. injected with AdVING4/p53 ( $1 \times 10^{8}$ GTU), AdVING4 (1x10 ${ }^{8}$ GTU), AdVp53 (1x10 $\left.{ }^{8} \mathrm{GTU}\right)$, AdV ( $\left.1 \times 10^{8} \mathrm{GTU}\right)$ or PBS every other day for a total 5 times. The tumor volume (B) was measured before and after treatment. The xenografted tumors were removed $(\mathrm{C})$ and tumor weight $(\mathrm{D})$ was measured 2 weeks after treatment. ${ }^{*} \mathrm{P}<0.05$ compared with $\mathrm{PBS}$ and AdV group; ${ }^{*} \mathrm{P}<0.05$ compared with AdVING4 and AdVp53 group, $\mathrm{Q}_{\text {volume }}=0.898,1.094,1.005,1.153,1.163$ and 1.183 at days $4,6,8,10,12$ and 14 after treatment, $Q_{\text {weight }}=1.197$, two-way and one-way repeated measures ANOVA and multiple comparisons; $n=5$ replicates per condition. Data shown are representative of three independent experiments.

(SD) and statistically processed by one-way and two-way repeated measures analysis of variance (ANOVA) and multiple comparisons using SPSS 10.0 software (SPSS, Chicago, IL, USA). A value of $\mathrm{P}<0.05$ was considered statistically significant. The interactive effect of ING4 and p53 was evaluated by Q-value calculated by the formula (35), Q=F(A+B)/FA+(1-FA) $\mathrm{FB}$, where $\mathrm{F}(\mathrm{A}+\mathrm{B})$ represents the fraction affected by treatment with AdVING4/p53 compared to the untreated control group, FA represents the fraction affected by AdVING4, and FB represents the fraction affected by AdVp53. A value of $\mathrm{Q}>1.15$ indicates a synergistic effect, $\mathrm{Q}<0.85$ indicates an antagonistic effect, and $\mathrm{Q}$ between 0.85 and 1.15 indicates an additive effect.

\section{Results}

Adenovirus-mediated ING4 andp53doublegene co-expression. To select an optimal MOI for a maximal adenovirus-mediated ING4 and/or p53 transgene expression but a minimal adenovirus itself-induced cytotoxic effect, the MDA-MB-231 human breast cancer cells were infected with marker gene GFP-expressing AdVING4/p53, AdVING4, AdVp53 or AdV at different MOIs for $24 \mathrm{~h}$, then the GFP expression was examined by fluorescence microscopy. More than $90 \%$ of GFP expression was found in AdVING4/p53-, AdVING4-, AdVp53- or AdV-infected MDA-MB-231 tumor cells at a MOI of 50 (Fig. 1B) or above (data not shown), whereas GFP expression was not found in uninfected MDA-MB-231 control tumor cells. Furthermore, there was almost no adenovirus-elicited cytotoxicity in 50 MOI blank adenovirus AdV-infected MDA-MB-231 tumor cells compared to control MDA-MB-231 tumor cells (Fig. 1B). RT-PCR (Fig. 1C) and western blot analysis (Fig. 1D) further demonstrated that adenovirus-mediated ING4 and/or p53 tumor suppressor gene was abundantly expressed at both the transcriptional and translational levels in 50 MOI AdVING4/p53-, AdVING4-, AdVp53-infected MDA-MB-231 tumor cells but not in AdV-infected and uninfected control cells. In addition, the endogenous mutant p53 was also obviously detected in AdV- or AdVING4-infected and uninfected control cells (Fig. 1C and D). These data indicated that 50 MOI can be employed as an optimal infection dose for adenovirus-mediated ING4 and p53 gene co-transfer in MDA-MB-231 tumor cells.

Synergistic tumor suppression by AdVING4/p53. To examine the combined effect of ING4 and p53 double tumor suppressors 
A
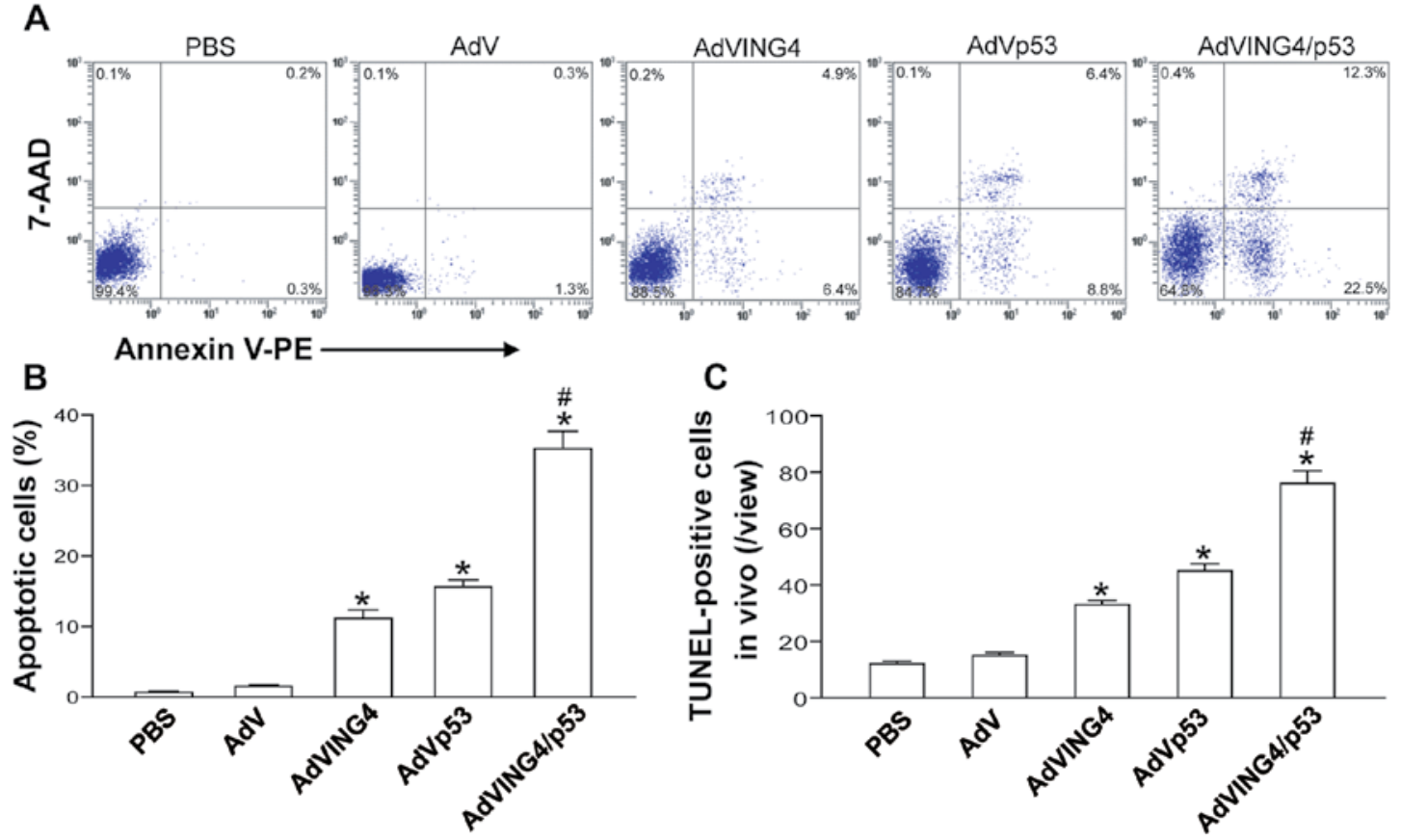

Figure 3. AdVING4/p53 induces synergistic tumor apoptosis in breast cancer. (A and B) Flow cytometric analysis of in vitro apoptosis. The MDA-MB-231 human breast cancer cells were treated with AdVING4/p53 (50 MOI), AdVING4 (50 MOI), AdVp53 (50 MOI), AdV (50 MOI) or PBS without adenovirus. Twenty-four hours after treatment, the tumor cells were harvested, stained with Annexin V-PE and 7-AAD, and then analyzed by flow cytometry. Representative flow cytometric images are shown (A). The Annexin V-positive cells including Annexin V single-positive cells (early apoptotic cells) and Annexin V/7-AAD double-positive cells (late apoptotic cells) in the total cell population represent apoptotic cells (B). ${ }^{*} \mathrm{P}<0.05$ compared with PBS and AdV group; ${ }^{\mathrm{P}}<0.05$ compared with AdVING4 and AdVp53 group, $\mathrm{Q}=1.447$, one-way repeated measures ANOVA and multiple comparisons; $\mathrm{n}=3$ replicates per condition. (C) TUNEL analysis of in vivo apoptosis. The TUNEL-positive cells represent in vivo apoptotic cells. "P<0.05 compared with PBS and AdV group; ${ }^{\text {"}} \mathrm{P}<0.05$ compared with AdVING4 and AdVp53 group, $\mathrm{Q}=1.360$, one-way repeated measures ANOVA and multiple comparisons; $\mathrm{n}=5$ replicates per condition, $\mathrm{n}=5$ sections per sample, $\mathrm{n}=5$ observations per section. Data shown are representative of three independent experiments.

on breast cancer in vitro, we performed AdVING4/p53-mediated gene co-transfer in MDA-MB-231 human breast cancer cells and assessed the MDA-MB-231 tumor cell viability by MTT assay. Compared with PBS and AdV control group, adenovirus-mediated ING4 and/or p53 expression significantly suppressed MDA-MB-231 tumor cell growth in vitro in a time-dependent manner with a peak inhibition at day 4 after infection (Fig. 2A, $\mathrm{P}<0.05$ ). Moreover, combination treatment by ING4 and 553 coexpression resulted in a synergistic growth inhibition in MDA-MB-231 tumor cells $(\mathrm{P}<0.05 ; \mathrm{Q}=1.189$, $1.158,1.168$ and 1.176 at days $1,2,3$ and 4 after infection, respectively). To further determine whether AdVING4/p53-mediated synergistic growth suppression observed in vitro could be reproduced in vivo, we evaluated combined effect of ING4 and p53 on MDA-MB-231 s.c. human breast cancer xenografted tumor growth in athymic nude mice. As shown in Fig. 2B-D, AdVING4/p53 also synergistically repressed MDA-MB-231 human breast cancer xenografted tumor growth in vivo $\left(\mathrm{P}<0.05 ; \mathrm{Q}_{\text {volume }}=0.898,1.094,1.005,1.153,1.163\right.$ and 1.183 at days $4,6,8,10,12$ and 14 after treatment, $\mathrm{Q}_{\text {weight }}=1.197$, respectively).

Synergistic induction of apoptosis by AdVING4/p53. To explore the cellular mechanism by which AdVING4/p53 synergistically inhibits tumor cell growth, apoptosis of MDA-MB-231 human breast cancer cells treated with AdVING4/p53, AdVING4, AdVp53 or AdV for $24 \mathrm{~h}$ and untreated control cells were analyzed using Annexin V-PE/7-AAD double staining by flow cytometry. As shown in Fig. 3A and B, treat- ment with AdVING4/p53, AdVING4 or AdVp53 remarkably induced 35.2, 11.2 and $15.6 \%$ apoptosis including early and late apoptotic cells in MDA-MB-231 tumor cells $(\mathrm{P}<0.05)$, whereas there were only 0.7 and $1.5 \%$ spontaneously apoptotic MDA-MB-231 tumor cells occurring in those grown in the medium containing PBS or AdV, respectively. Furthermore, AdVING4/p53 more efficiently triggered MDA-MB-231 breast cancer cell apoptosis with a synergistic effect $(\mathrm{P}<0.05$; $\mathrm{Q}=1.447)$. To confirm AdVING4/p53-induced apoptotic effect in vivo, we further assessed the apoptosis in MDA-MB-231 human breast cancer s.c. xenografted tumors by TUNEL assay (Fig. 3C). Consistent with the results in vitro by flow cytometric analysis of apoptosis, AdVING4/p53 also has a synergistic efficacy for in vivo inducing MDA-MB-231 breast tumor apoptosis in athymic nude mice $(\mathrm{P}<0.05 ; \mathrm{Q}=1.360)$.

Enhanced p53 acetylation and transcriptional activity by ING4. To elucidate the molecular mechanism responsible for AdVING4/p53 combined gene therapy-induced synergistic antitumor effect, the expression of tumor suppressor p53/Ac-p53 (K382), Cip/Kip family cyclin-dependent kinase inhibitor (CDI) P21 and apoptosis-related proteins such as Bax, PUMA, Noxa, Bcl-2, caspase-9, caspase-3 and PARP in MDA-MB-231 human breast cancer cells after different treatment were analyzed by western blot analysis. As shown in Fig. 4A, the p53 and Ac-p53 (K382) was substantially increased in AdVp53 and AdVING4/p53 group. Additionally, the P21, Bax, PUMA, Noxa, cleaved caspase-9, cleaved caspase-3 and cleaved PARP in AdVING4, AdVp53 and AdVING4/p53 group 
A

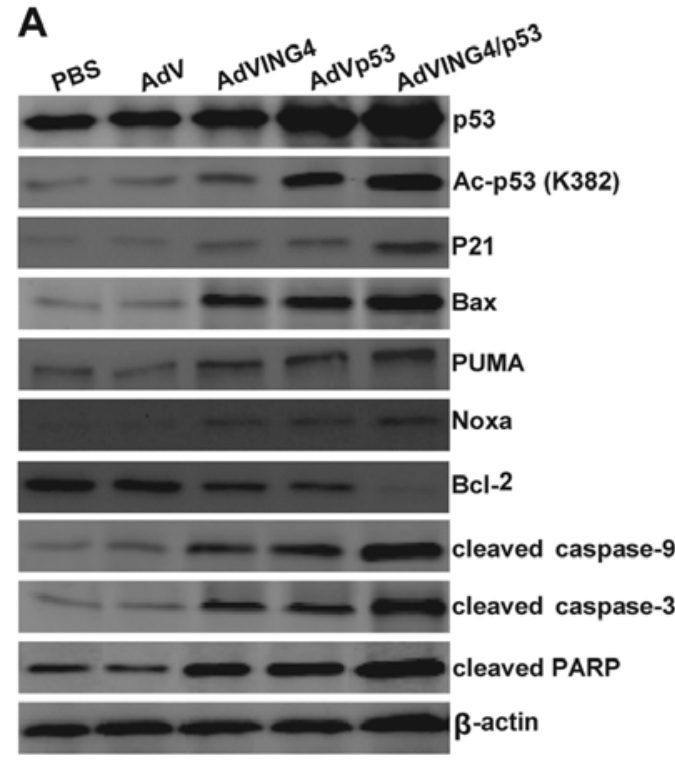

B

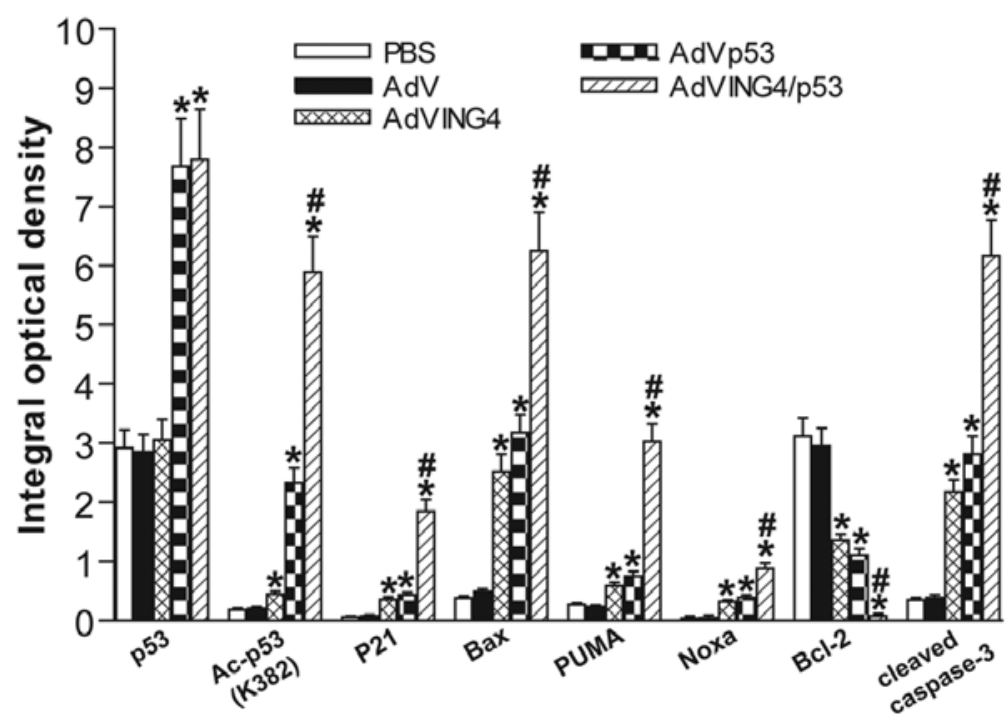

Figure 4. AdVING4/p53 enhances upregulation of cyclin-dependent kinase inhibitor and activation of intrinsic apoptosis via ING4-mediated enhancement of p53 acetylation and transcriptional responses. (A) Western blot analysis of cyclin-dependent kinase inhibitor and apoptosis-related proteins. The total cellular lysates derived from AdVING4/p53-, AdVING4-, AdVp53-, AdV- or PBS-treated MDA-MB-231 human breast cancer cells were immunoblotted with a panel of antibodies specific for p53, Ac-p53 (K382), P21, Bax, PUMA, Noxa, Bcl-2, caspase-9, caspase-3, PARP and $\beta$-actin (an internal control), respectively. The representative pictures of western blot analysis are shown. (B) Immunohistochemistry analysis of cyclin-dependent kinase inhibitor and apoptosis-related proteins in MDA-MB-231 s.c. xenografted tumors. The immunostaining intensity of p53, Ac-p53 (K382), P21, Bax, PUMA, Noxa, Bcl-2 and cleaved caspase-3 was quantified as integral optical density (IOD) by Image-Pro Plus 6.0 software. " $\mathrm{P}<0.05$ compared with PBS and AdV group; ${ }^{*} \mathrm{P}<0.05$ compared with AdVING4 and AdVp53 group, $\mathrm{Q}_{\mathrm{Ac}-\mathrm{p} 53}=2.365, \mathrm{Q}_{\mathrm{P} 21}=2.676, \mathrm{Q}_{\mathrm{Bax}}=1.203, \mathrm{Q}_{\mathrm{PUMA}}=3.444, \mathrm{Q}_{\mathrm{Noxa}}=1.335, \mathrm{Q}_{\mathrm{Bcl}-2}=1.153, \mathrm{Q}_{\text {cleaved caspase-3}}=1.366$, one-way repeated measures ANOVA and multiple comparisons; $\mathrm{n}=5$ replicates per condition, $\mathrm{n}=5$ sections per sample, $\mathrm{n}=5$ observations per section. Data shown are representative of three independent experiments.

was remarkably increased, whereas the Bcl-2 was significantly decreased. Moreover, AdVING4/p53 resulted in an enhanced effect on upregulation of Ac-p53 (K382) and p53-downstream genes such as P21, Bax, PUMA and Noxa, activation of caspase-9, caspase-3 and cleavage of PARP as well as downregulation of Bcl-2. The combined effect of AdVING4/p53 on in vivo expression of p53, Ac-p53 (K382), P21, Bax, PUMA, Noxa, Bcl-2, cleaved caspase-3 in MDA-MB-231 human breast cancer s.c. xenografted tumors was further confirmed by immunohistochemistry analysis (Fig. 4B).

Synergistic inhibition of in vivo tumor angiogenesis by AdVING4/p53. To investigate the combined effect of AdVING4/p53 on tumor angiogenesis in vivo, the microvessel density (MVD) in MDA-MB-231 human breast cancer s.c. xenografted tumors was detected by CD31 immunohistochemistry analysis. The positive expression of CD31 was mainly presented as brownish yellow or brownish granules in tumor vascular endothelial cells of MDA-MB-231 xenografted tumors (Fig. 5A). Compared with PBS- and AdV-treated control group, the CD31 expression in AdVING4-, AdVp53- and AdVING4/p53-treated group was weaker or less (Fig. 5A and B, $\mathrm{P}<0.05$ ). In addition, the MVD counted in AdVING4, AdVp53 and AdVING4/p53 group was significantly less than that in PBS and AdV group (Fig. 5C, P<0.05). Furthermore, AdVING4/p53 combined gene therapy has a synergistic effect on downregulation of CD31 and reduction of MVD in MDA-MB-231 breast cancer xenografted tumors in athymic nude mice $(\mathrm{P}<0.05$; $\mathrm{QCD} 31=1.185$ and $\mathrm{QMVD}=1.176)$.

\section{Discussion}

It is widely accepted that p53 is an important molecular target in human cancers and restoring wild-type p53 function would provide an effective alternative for cancer therapy. Post-translational modifications of p53 such as phosphorylation, ubiquitination, methylation, sumoylation, neddylation and acetylation are crucial for its activation and determining p53-dependent cellular outcomes $(1,36)$. Among them, acetylation is indispensable for p53 activation (31). It has also been reported that ING4 as a novel candidate tumor suppressor can physically interact with p53 and p300 in the nucleus and consequently enhance p53 acetylation and its transcriptional activity via its bipartite nuclear localization signal (NLS) domain $(10,37)$. In the study, we examined the combined effects of p53 and ING4 tumor suppressors on MDA-MB-231 breast cancer cells in vitro and in vivo in an athymic nude mouse model by adenovirusmediated ING4 and p53 co-expression (AdVING4/p53). We demonstrated that AdVING4/p53 induced in vitro synergistic growth inhibition and apoptosis in p53-mutant MDA-MB-231 human breast cancer cells. Moreover, AdVING4/p53 treatment also synergistically suppressed in vivo breast cancer growth and induced apoptosis in MDA-MB-231 s.c. xenografted tumors in athymic nude mice.

To delineate the molecular mechanism underlying AdVING4/p53-mediated synergistic antitumor effects, the expression of cell cycle- and apoptosis-related molecules such as p53, Ac-p53 (K382), P21, Bax, PUMA, Noxa, Bcl-2, caspase-9, caspase-3 and PARP in MDA-MB-231 breast 

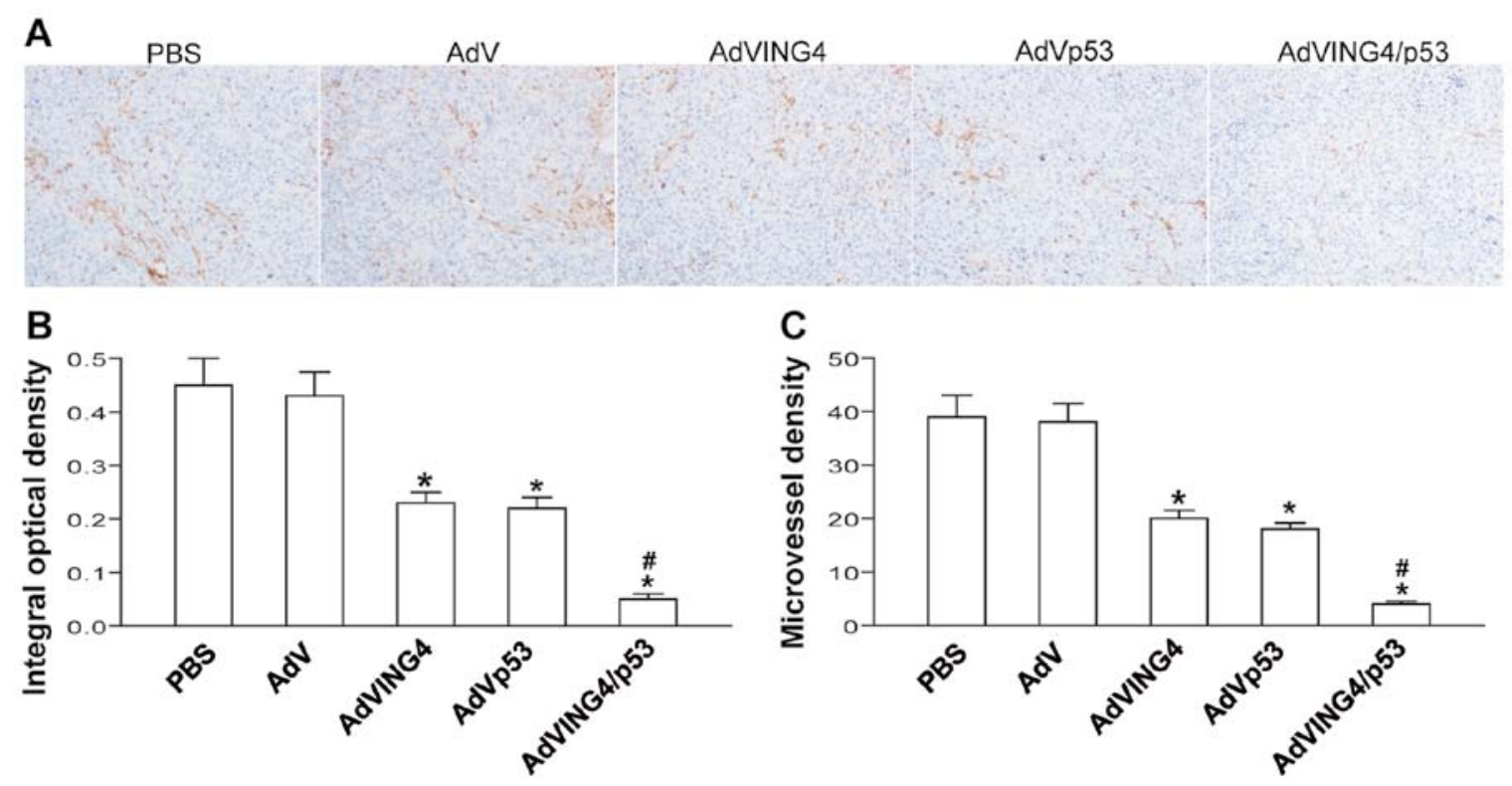

Figure 5. AdVING4/p53 synergistically suppresses breast tumor angiogenesis in vivo. (A) Representative pictures of immunohistochemical detection of CD31 in MDA-MB-231 human breast cancer s.c. xenografted tumors. (B) The integral optical density (IOD) of CD31 immunostaining quantified by Image-Pro Plus 6.0 software. "P<0.05 compared with PBS and AdV group; ${ }^{~} \mathrm{P}<0.05$ compared with AdVING4 and AdVp53 group, $\mathrm{Q}=1.185$, one-way repeated measures ANOVA and multiple comparisons; $n=5$ replicates per condition, $n=5$ sections per sample, $n=5$ observations per section. (C) The microvessel density (MVD) in MDA-MB-231 xenografted tumors. " $\mathrm{P}<0.05$ compared with PBS and AdV group; ${ }^{*} \mathrm{P}<0.05$ compared with AdVING4 and AdVp53 group, $\mathrm{Q}=1.176$, one-way repeated measures ANOVA and multiple comparisons; $n=5$ replicates per condition, $n=5$ sections per sample, $n=5$ observations per section. Data shown are representative of three independent experiments.

cancer cells in vitro and in vivo xenografted tumors after different treatment was analyzed by western blot analysis and/or immunohistochemistry. The cyclin-dependent kinase (CDK) inhibitor P21 and pro-apoptosis proteins such as Bax, Puma and Noxa are the well-characterized p53 target genes involved in cell cycle arrest and apoptosis (2). P21 as an important member of CDK inhibitor Cip/Kip family can prevent the activation of cyclin E/A-CDK2 complex and induce cell cycle G1 phase arrest (38). Bcl-2 family is a key regulator of apoptosis and a crucial determinant of cell fate (39). The ratio of pro- to anti-apoptotic molecules such as Bax/Bcl-2 constitutes a rheostat that sets the threshold of susceptibility to apoptosis for the intrinsic pathway (39), including pore formation in mitochondrial outer membrane, loss of mitochondrial integrity, release of cytochrome $c$ into the cytosol, activation of caspase- 9 , caspase- 3 and cleavage of PARP. In addition, PUMA and Noxa BH3-only pro-apoptosis proteins can bind to Bcl-2 anti-apoptosis protein in the mitochondria and promote cytochrome $c$ release, leading to intrinsic apoptosis (39). In the present study, we found that the levels of Ac-p53, P21, Bax, PUMA, Noxa, cleaved caspase-9, cleaved caspase-3 and cleaved PARP was significantly upregulated in AdVp53-treated MDA-MB-231 tumor cells, whereas the Bcl-2 was downregulated. The increased Ac-p53 in AdVp53 group may be accompanied by the adenovirus-mediated increased synthesis of p53. In addition, AdVING4 single gene therapy also markedly increased the P21, Bax, PUMA, Noxa, cleaved caspase-9, cleaved caspase- 3 and cleaved PARP while decreased the Bcl-2 in p53-mutant MDA-MB-231 tumor cells, which may be elicited by p53-independent mechanisms such as other transcription factors involved and chromatin remolding $(12,40)$. Interestingly, AdVING4/p53 combined therapy resulted in an enhanced effect on upregulation of Ac-p53 and p53-downstream genes such as P21, Bax, PUMA and Noxa, downregulation of Bcl-2, activation of caspase-9, caspase-3 and cleavage of PARP to a great extent via ING4-induced enhancement of p53 acetylation and its transcriptional responses, which may be an important mechanism accountable for AdVING4/p53-induced synergistic growth suppression and apoptosis in MDA-MB-231 breast cancer cells.

Tumor angiogenesis is a hallmark of cancer, which is governed by two countervailing factors that either facilitate or oppose angiogenesis (41). It has been shown to be indispensable for progressive tumor growth and metastasis, representing a potential therapeutic target in anticancer therapy (41). A great deal of data suggested that tumor angiogenesis inhibition and vessel normalization is a promising and non-toxic anticancer therapeutic strategy $(42,43)$. It has been reported that p53 can inhibit tumor angiogenesis by downregulating pro-angiogenic factor VEGF expression via MDM2-mediated HIF-1 $\alpha$ degradation (44) and miR-107-induced HIF-1 $\beta$ suppression (45) as well as by upregulating production of anti-angiogenic collagen fragments via promoting collagen prolyl hydroxylase expression (46). It has also been shown that ING4 can repress expression of pro-angiogenic factors such as IL-6 and IL-8 via inhibiting transcriptional activity of NF- $\mathrm{\kappa B}$ and HIF- $1 \alpha$, leading to the inhibition of tumor angiogenesis $(22,25)$. To investigate the combined effect of AdVING4/p53 on tumor angiogenesis in vivo, the MVD in MDA-MB-231 human breast cancer s.c. xenografted tumor tissues was evaluated by CD31 immunohistochemistry analysis. Our data showed that AdVING4/p53 synergistically downregulated CD31 expression and decreased MVD in MDA-MB-231 breast cancer 
xenografted tumors, which may be another important mechanism involved in AdVING4/p53-mediated in vivo synergistic growth inhibition in human breast cancer xenografted tumors in athymic nude mice.

In summary, adenovirus-mediated p53 and ING4 combined gene therapy induced synergistic growth inhibition and apoptosis as well as enhanced effects on upregulation of acetylated p53, P21, Bax, PUMA, Noxa, cleaved caspase-9, cleaved caspase-3 and cleaved PARP, and downregulation of Bcl-2, CD31 and microvessel density (MVD) in MDA-MB-231 breast cancer in vitro and/or in vivo subcutaneous (s.c.) xenografted tumors. The synergistic antitumor activity elicited by AdVING4/p53 was closely associated with the enhanced activation of intrinsic apoptotic pathway and synergistic inhibition of tumor angiogenesis, very possibly via ING4-mediated enhancement of p53 acetylation and activity. Thus, out results indicate that cancer gene therapy combining two or more tumor suppressors such as p53 and ING4 may constitute a novel and effective therapeutic modality for human breast cancer and other cancers.

\section{Acknowledgements}

This research study was supported by grants from the National Natural Science Foundation of China (NNSFC) (nos. 81372443, 81001016, 81272542 and 81572992) and the Science and Technology Department of Jiangsu Province (nos. BL2014039 and BY2015039).

\section{References}

1. Kruse JP and Gu W: Modes of p53 regulation. Cell 137: 609-622, 2009.

2. Wei CL, Wu Q, Vega VB, Chiu KP, Ng P, Zhang T, Shahab A, Yong $\mathrm{HC}, \mathrm{Fu} \mathrm{Y}$, Weng Z, et al: A global map of p53 transcription-factor binding sites in the human genome. Cell 124: 207-219, 2006.

3. Kandoth C, McLellan MD, Vandin F, Ye K, Niu B, Lu C, Xie M, Zhang Q, McMichael JF, Wyczalkowski MA, et al: Mutational landscape and significance across 12 major cancer types. Nature 502: 333-339, 2013

4. Muller PA and Vousden KH: Mutant p53 in cancer: New functions and therapeutic opportunities. Cancer Cell 25: 304-317, 2014

5. Muller PA and Vousden KH: p53 mutations in cancer. Nat Cell Biol 15: 2-8, 2013

6. Tchelebi L, Ashamalla H and Graves PR: Mutant p53 and the response to chemotherapy and radiation. Subcell Biochem 85 133-159, 2014.

7. Cheok CF, Verma CS, Baselga J and Lane DP: Translating p53 into the clinic. Nat Rev Clin Oncol 8: 25-37, 2011.

8. Lane DP, Cheok CF and Lain S: p53-based cancer therapy. Cold Spring Harb Perspect Biol 2: a001222, 2010.

9. Tallen G and Riabowol K: Keep-ING balance: Tumor suppression by epigenetic regulation. FEBS Lett 588: 2728-2742, 2014.

10. Shiseki M, Nagashima M, Pedeux RM, Kitahama-Shiseki M, Miura K, Okamura S, Onogi H, Higashimoto Y, Appella E, Yokota J, et al: p29ING4 and p28ING5 bind to p53 and p300, and enhance p53 activity. Cancer Res 63: 2373-2378, 2003.

11. Zhang $X, X u$ LS, Wang ZQ, Wang KS, Li N, Cheng ZH, Huang SZ, Wei DZ and Han ZG: ING4 induces G2/M cell cycle arrest and enhances the chemosensitivity to DNA-damage agents in HepG2 cells. FEBS Lett 570: 7-12, 2004.

12. Unoki M, Shen JC, Zheng ZM and Harris CC: Novel splice variants of ING4 and their possible roles in the regulation of cell growth and motility. J Biol Chem 281: 34677-34686, 2006.

13. Xie Y, Zhang H, Sheng W, Xiang J, Ye Z and Yang J: Adenovirus-mediated ING4 expression suppresses lung carcinoma cell growth via induction of cell cycle alteration and apoptosis and inhibition of tumor invasion and angiogenesis. Cancer Lett 271: $105-116,2008$
14. Cai L, Li X, Zheng S, Wang Y, Wang Y, Li H, Yang J and Sun J: Inhibitor of growth 4 is involved in melanomagenesis and induces growth suppression and apoptosis in melanoma cell line M14. Melanoma Res 19: 1-7, 2009.

15. Gong A, Ye S, Xiong E, Guo W, Zhang Y, Peng W, Shao G, Jin J, Zhang Z, Yang J, et al: Autophagy contributes to ING4-induced glioma cell death. Exp Cell Res 319: 1714-1723, 2013.

16. Xie Y, Sheng W, Miao J, Xiang J and Yang J: Enhanced antitumor activity by combining an adenovirus harboring ING4 with cisplatin for hepatocarcinoma cells. Cancer Gene Ther 18: 176-188, 2011.

17. Zhao Y, Su C, Zhai H, Tian Y, Sheng W, Miao J and Yang J: Synergistic antitumor effect of adenovirus-mediated hING4 gene therapy and (125)I radiation therapy on pancreatic cancer. Cancer Lett 316: 211-218, 2012.

18. Ling C, Xie Y, Zhao D, Zhu Y, Xiang J and Yang J: Enhanced radiosensitivity of non-small-cell lung cancer (NSCLC) by adenovirus-mediated ING4 gene therapy. Cancer Gene Ther 19: 697-706, 2012.

19. Kim S, Chin K, Gray JW and Bishop JM: A screen for genes that suppress loss of contact inhibition: Identification of ING4 as a candidate tumor suppressor gene in human cancer. Proc Natl Acad Sci USA 101: 16251-16256, 2004.

20. Shen JC, Unoki M, Ythier D, Duperray A, Varticovski L, Kumamoto K, Pedeux R and Harris CC: Inhibitor of growth 4 suppresses cell spreading and cell migration by interacting with a novel binding partner, liprin alpha1. Cancer Res 67: 2552-2558, 2007.

21. Li J, Martinka M and Li G: Role of ING4 in human melanoma cell migration, invasion and patient survival. Carcinogenesis 29: 1373-1379, 2008

22. Garkavtsev I, Kozin SV, Chernova O, Xu L, Winkler F, Brown E, Barnett GH and Jain RK: The candidate tumour suppressor protein ING4 regulates brain tumour growth and angiogenesis. Nature 428: 328-332, 2004

23. Nozell S, Laver T, Moseley D, Nowoslawski L, De Vos M, Atkinson GP, Harrison K, Nabors LB and Benveniste EN: The ING4 tumor suppressor attenuates NF-kappaB activity at the promoters of target genes. Mol Cell Biol 28: 6632-6645, 2008.

24. Ozer A, Wu LC and Bruick RK: The candidate tumor suppressor ING4 represses activation of the hypoxia inducible factor (HIF). Proc Natl Acad Sci USA 102: 7481-7486, 2005.

25. Colla S, Tagliaferri S, Morandi F, Lunghi P, Donofrio G, Martorana D, Mancini C, Lazzaretti M, Mazzera L, Ravanetti L, et al: The new tumor-suppressor gene inhibitor of growth family member 4 (ING4) regulates the production of proangiogenic molecules by myeloma cells and suppresses hypoxia-inducible factor-1 alpha (HIF-1alpha) activity: Involvement in myeloma-induced angiogenesis. Blood 110: 4464-4475, 2007.

26. Hou Y, Zhang Z, Xu Q, Wang H, Xu Y and Chen K: Inhibitor of growth 4 induces NFאB/p65 ubiquitin-dependent degradation. Oncogene 33: 1997-2003, 2014

27. Lu M, Pan C, Zhang L, Ding C, Chen F, Wang Q, Wang K and Zhang X: ING4 inhibits the translation of proto-oncogene MYC by interacting with AUF1. FEBS Lett 587: 1597-1604, 2013.

28. Jemal A, Bray F, Center MM, Ferlay J, Ward E and Forman D: Global cancer statistics. CA Cancer J Clin 61: 69-90, 2011.

29. Brenner MK, Gottschalk S, Leen AM and Vera JF: Is cancer gene therapy an empty suit? Lancet Oncol 14: e447-e456, 2013.

30. Wilson DR: Viral-mediated gene transfer for cancer treatment. Curr Pharm Biotechnol 3: 151-164, 2002.

31. Tang Y, Zhao W, Chen Y, Zhao Y and Gu W: Acetylation is indispensable for $\mathrm{p53}$ activation. Cell 133: 612-626, 2008.

32. Xie Y, Lv H, Sheng W, Miao J, Xiang J and Yang J: Synergistic tumor suppression by adenovirus-mediated inhibitor of growth 4 and interleukin-24 gene cotransfer in hepatocarcinoma cells. Cancer Biother Radiopharm 26: 681-695, 2011.

33. Liu Y, Ye T, Sun D, Maynard J and Deisseroth A: Conditionally replication-competent adenoviral vectors with enhanced infectivity for use in gene therapy of melanoma. Hum Gene Ther 15: 637-647, 2004.

34. He TC, Zhou S, da Costa LT, Yu J, Kinzler KW and Vogelstein B: A simplified system for generating recombinant adenoviruses. Proc Natl Acad Sci USA 95: 2509-2514, 1998.

35. Wang W, Qin SK, Chen BA and Chen HY: Experimental study on antitumor effect of arsenic trioxide in combination with cisplatin or doxorubicin on hepatocellular carcinoma. World J Gastroenterol 7: 702-705, 2001.

36. Brooks $\mathrm{CL}$ and $\mathrm{Gu} \mathrm{W}$ : Ubiquitination, phosphorylation and acetylation: The molecular basis for p53 regulation. Curr Opin Cell Biol 15: 164-171, 2003. 
37. Zhang X, Wang KS, Wang ZQ, Xu LS, Wang QW, Chen F, Wei DZ and Han ZG: Nuclear localization signal of ING4 plays a key role in its binding to p53. Biochem Biophys Res Commun 331: 1032-1038, 2005.

38. Massagué J: G1 cell-cycle control and cancer. Nature 432: 298-306, 2004

39. Danial NN and Korsmeyer SJ: Cell death: Critical control points. Cell 116: 205-219, 2004.

40. Hung T, Binda O, Champagne KS, Kuo AJ, Johnson K, Chang HY, Simon MD, Kutateladze TG and Gozani O: ING4 mediates crosstalk between histone $\mathrm{H} 3 \mathrm{~K} 4$ trimethylation and $\mathrm{H} 3$ acetylation to attenuate cellular transformation. Mol Cell 33: 248-256, 2009.

41. Hanahan D and Weinberg RA: Hallmarks of cancer: The next generation. Cell 144: 646-674, 2011

42. Welti J, Loges S, Dimmeler S and Carmeliet P: Recent molecular discoveries in angiogenesis and antiangiogenic therapies in cancer. J Clin Invest 123: 3190-3200, 2013.
43. Shang B, Cao Z and Zhou Q: Progress in tumor vascular normalization for anticancer therapy: Challenges and perspectives. Front Med 6: 67-78, 2012.

44. Ravi R, Mookerjee B, Bhujwalla ZM, Sutter CH, Artemov D, Zeng Q, Dillehay LE, Madan A, Semenza GL and Bedi A: Regulation of tumor angiogenesis by p53-induced degradation of hypoxia-inducible factor 1alpha. Genes Dev 14: 34-44, 2000.

45. Yamakuchi M, Lotterman CD, Bao C, Hruban RH, Karim B, Mendell JT, Huso D and Lowenstein CJ: P53-induced microRNA-107 inhibits HIF-1 and tumor angiogenesis. Proc Natl Acad Sci USA 107: 6334-6339, 2010.

46. Teodoro JG, Parker AE, Zhu X and Green MR: p53-mediated inhibition of angiogenesis through up-regulation of a collagen prolyl hydroxylase. Science 313: 968-971, 2006. 